Die Geschichte des Zürcher Medizinstudiums steht im Mittelpunkt des Sammelbands «Innenansichten einer Ärzteschmiede», der anlässlich des Universitätsjubiläums im Chronos-Verlag erschienen ist. Die Schweizerische Ärztezeitung druckt Auszüge aus den Artikeln in einer Serie ab. Unter dem Titel «Vom Grünschnabel zum Weisskittel» zeigt das Medizinhistorische Archiv zudem bis 31. Mai 2008 eine Ausstellung zum Thema im Hauptgebäude der Universität (www.175jahre.uzh.ch/gruenschnabel-weisskittel).

Der Beitrag, aus dem die heutigen Auszüge stammen, handelt vom Psychiatrieprofessor Auguste Forel, der den Hypnotismus an der Universität Zürich in die Medizinerausbildung einführte. Das als «Königsweg zur Psyche» angesehene Verfahren war für Forel indes auch ein Instrument, an seinem Traum einer lenkbaren Gesellschaft zu arbeiten.

\title{
Auguste Forels Hypnotismus im Vorlesungssaal und in der Klinik*
}

\author{
Mirjam Bugmann
}

\footnotetext{
* Auszug aus dem Beitrag der Autorin in: Ritzmann I, Schweer W, Wolff E (Hrsg.). Innenansichten einer Ärzteschmiede. Lehren, lernen und leben - aus der Geschichte des Zürcher Medizinstudiums. Zürich: Chronos; 2008. 240 Seiten, 80 teils farbige Abbildungen. Fr. 34.- $€ 20.60$. ISBN 978-3-0340-0909-6. Quellenverweise im Originalartikel.
}

Korrespondenz:

lic. phil. Mirjam Bugmann Universität Zürich

Forschungsstelle für Sozialund Wirtschaftsgeschichte Rämistrasse 64

CH-8001 Zürich

mbugmann@vtxmail.ch
Sommersemester 1896, Samstag, 14.30 Uhr im Konzert- und Kliniksaal des Burghölzli. Die Studierenden des praktischen Kurses über Suggestion und Suggestivtherapie warten gespannt auf die Patienten, die Professor Forel ihnen heute vorstellen und vor ihren Augen nach Präsentation der Krankengeschichte hypnotisieren wird. «Dem Auditorium gegenüber sind in einem Halbkreis bequeme Fauteuils und Polsterbänke aufgestellt, auf welchen die zu hypnotisirenden Leute Platz nehmen. In ihrer Mitte sitzt der Vortragende; die Patienten kommen von beiden Seiten her durch Seitenthüren herein, von der einen Seite die Männer, von der anderen die Frauen, und werden im Nebenzimmer jeweils vor der Hypnose untersucht und befragt.»

Hereingeführt wird Lina F., 40jährig, «erblich beträchtlich belastet», wie Forel einleitend anmerkt. Die Frau zeigt seit drei Jahren Zeichen zunehmender Nervosität und wird von religiösen Zweifeln gequält. Aufgrund der depressiven Stimmung und heftiger Schmerzen am Ellenbogen verlangt die Patientin die Versetzung ins Burghölzli zwecks Hypnosebehandlung. Forel gibt den studierenden Hypnosenovizen klare Regieanweisungen: «Dem zu Hypnotisierenden trete man [...] ganz natürlich und zielbewusst gegenüber, erkläre ihm, es sei nichts Unnatürliches, nichts Zauberhaftes, sondern eine einfache, jedem Menschen zukommende Eigenschaft des Nervensystems, und er werde ganz gut beeinflusst werden [...]. Man vermeide viele Worte und Erklärungen und setze den Patienten oder Nichtpatienten auf einen bequemen Lehnstuhl.»Der Psychiatrieprofessor stellt sich vor F. hin und schaut ihr konzentriert in die Augen. «Das geht ja ganz famos; Ihre Augen sind schon ganz feucht und Ihre

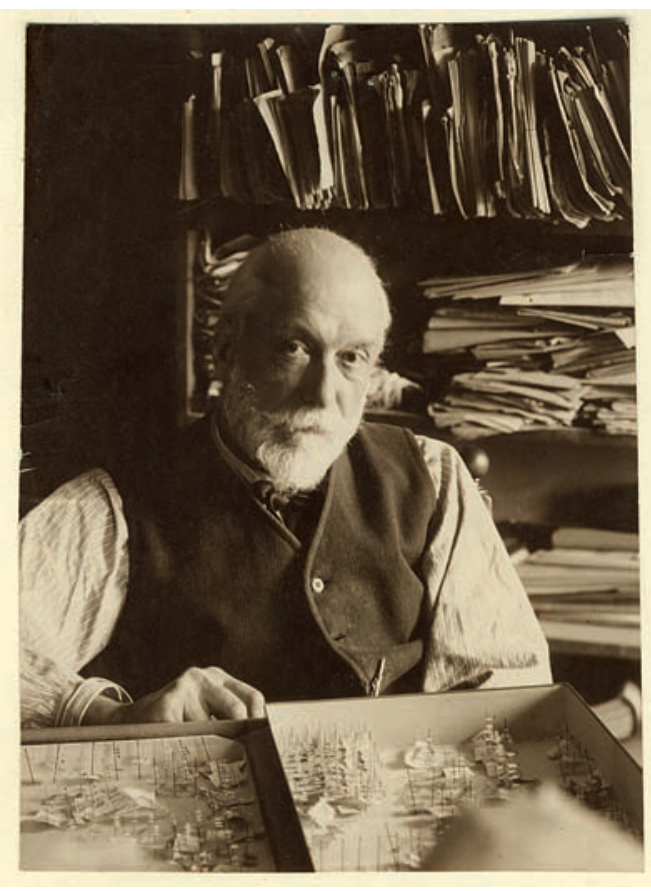

Auguste Forel präsentiert seine Forschungen, um 1908. Medizinhistorisches Archiv, Universität Zürich.

Lider schwer. Schauen Sie auf meine Finger!» Forel senkt die Finger, die Lider F.s schliessen sich. Die für die Hypnose gut empfängliche Patientin schläft. «Sie werden sich besser fühlen. Die Schmerzen im Arm verschwinden und Ihre religiösen Zweifel werden Sie verlassen.» Volontärarzt Bauer vermeldet den Therapieerfolg: «Als wir die Patientin nach dreimonatigem Aufenthalt entliessen, war ihre Gemüthsstimmung eine recht gute [...]. Über Schmerzen im Arm klagte die Patientin nun nicht mehr. Die religiösen Ideen waren ganz zurückgetreten. [...] Sie verliess 
die Anstalt mit dem Gefühl grosser Dankbarkeit, jedoch, wenn auch wesentlich gebessert, so doch nicht völlig geheilt.»

Seit dem Wintersemester 1887/88 führte Auguste Forel (1848-1931) Vorlesungen und klinische Demonstrationen zum Hypnotismus im Lehrangebot. Das Interesse an seinen Vorlesungen war gross. Wie Forel in seiner Autobiographie berichtet, sprengte der Zuschauerandrang die Kapazitäten des grössten Auditoriums, so dass er die Zulassung auf Medizin- und Jusstudenten beschränkte. Forel war zu dieser Zeit noch kein «alter Hase» in der Anwendung des Hypnotismus. Im März 1887 war er zu Hippolyte Bern-

\section{Möwenmärchen}

Harriet Ines Keller-Wossidlo

Korrespondenz:

Dr. med. Harriet Keller-Wossidlo

Klinik Barmelweid

CH-5017 Barmelweid

harriet.keller@swissonline.ch
Es war einmal eine wunderschöne Möwe. Sie war flecklos weiss, keine Feder war weniger weiss als die andere. In ihrer makellosen Schönheit strahlte sie und leuchtete - den Menschen zum Wohlgefallen. «Rührt meine Schönheit nicht an», schien sie mit klugen Augen zu sagen. Und aufmerksam hob sie den Kopf und beobachtete die Welt ...

Gerne sass sie auf einem Felsenvorsprung am Meer, das sich hier in sanften Buchten verlor. Nun kam der Tag, an dem sie ein ebenso reines Möwenbaby bekam. Auch das Möwenbaby hatte keine einzige graue Feder, und es war kräftig und fein zugleich.

Ein Mensch wollte diese weissen Federn zum eigenen Schmuck. Doch der Mensch wusste nicht, dass diese Möwen heilig waren und nicht berührt werden durften - obwohl er es hätte wissen müssen. Da das Möwenbaby noch feinere Federn hatte, raubte der Mensch der weissen Möwe ihr Baby. Die Möwenmutter stiess Warnrufe aus, um den Menschen vom Raub abzuhalten. Aber der Mensch verstand die Rufe der Möwin nicht, und er wollte sie auch nicht hören. Er nahm das Möwenbaby und ging fort. heim (1840-1919) - einem der damals bekanntesten Hypnotismusexponenten - nach Nancy gefahren, um mehr über den Hypnotismus zu erfahren und die neue Therapieform zu erlernen. Forel staunte: «Ich konnte während der fünf Tage nicht genug mich über alles Gesehene wundern.» Wieder zurück in Zürich wandte er die neu erworbene Kunst ausgiebig bei Patienten und Personal des Burghölzli an und nahm die Lehrtätigkeit zum Thema auf.

In der nächsten Folge: Degeneration und Eugenik in Zürcher pädiatrischen Lehrmitteln.
Die Möwenmutter rief und rief nach dem geraubten Baby, doch kein Mensch hörte ihre Klagen ... Was sollte sie tun, damit in Zukunft die Menschen die Möwennot verstehen und nie wieder ein Möwenbaby geraubt wird?

Der Menschräuber hatte auch ein Baby, und eines Morgens in der Früh, bei aufgehender Sonne, flog die Möwin und holte sich das Menschenbaby zu ihrem Felsen im Meereswasser. Das Menschenbaby schrie jämmerlich: kurze klagende Laute und langgezogene Rufe. Die Möwenmutter jedoch verstand die Not des Menschenkindes und brachte es zurück.

Damit aber die Menschen ewig an ihre Schandtat erinnert werden, gab die kluge weisse Möwe allen nachfolgenden Möwen als ihre Sprache diese menschlichen Klagelaute bis zum Ende eines Möwenlebens.

Die Moral von der Geschicht': «Hörst du die Rufe einer Möwe, vergiss das Menschenunrecht nicht.» 